\title{
Clinical significance of circulating galectins as colorectal cancer markers
}

\author{
MAKOTO WATANABE ${ }^{1,4}$, ICHIRO TAKEMASA $^{2}$, NAOKI KANEKO $^{1,4}$, YUHKI YOKOYAMA ${ }^{3}$, EI-ICHI MATSUO ${ }^{1,4}$, \\ SUSUMU IWASA $^{5}$, MASAKI MORI ${ }^{2}$, NARIAKI MATSUURA ${ }^{2,3}$, MORITO MONDEN $^{3}$ and OSAMU NISHIMURA ${ }^{1}$ \\ ${ }^{1}$ Division of Disease Proteomics, Institute for Protein Research; Departments of ${ }^{2}$ Gastroenterological Surgery, \\ Graduate School of Medicine and ${ }^{3}$ Pathology, School of Allied Health Science, Faculty of Medicine, Osaka University, \\ Osaka; ${ }^{4}$ Life Science Research Center, Technology Research Laboratory and ${ }^{5}$ Life Science Business Department, \\ Analytical and Measuring Instruments Division, Shimadzu Corporation, Kyoto, Japan
}

Received December 17, 2010; Accepted January 12, 2011

DOI: 10.3892/or.2011.1198

\begin{abstract}
The utility of CEA and CA19-9 as colorectal carcinoma (CRC) markers is limited and development of additional reliable markers is under investigation. We previously showed that galectin-1 is overexpressed in CRC tissues. If such a protein leaks into the peripheral circulation, it might constitute a tumor marker candidate. Here, we test the hypothesis that the levels of circulating galectins could reflect the presence of CRC and/or its progression state. We constructed sandwich ELISAs for galectin-1/-2/-3/-4/-7 and determined their plasma concentrations in $105 \mathrm{CRC}$ patients and 100 healthy volunteers (control). Matched pair samples of 56 patients pre- and post-surgery were also subjected to ELISA analysis. Circulating levels of galectin-1/-3/-4 in CRC patients were significantly higher compared to those in controls. Galectin-1 and galectin-4 levels significantly decreased after surgery $(\mathrm{P}<0.01)$, and the level of galectin- 4 in most patients fell below the cut-off value. The levels of circulating galectin- 4 significantly increased as the tumor stage progressed $(\mathrm{P}<0.001)$, whereas those for galectin-1 were relatively high from an early stage. Combined use of galectin-4 with CEA and/or CA19-9 markedly increased the proportion of CRC patients who were positive for tumor markers (from 33.3 to $59.0 \%$ for CEA and from 17.1 to $51.4 \%$ for CA19-9). Our data show that galectin-4 may be a tumor marker for use in patient follow-up, while galectin-1 could be used for tumor screening. In particular, galectin- 4 can be useful as a complementary marker when combined with CEA/CA19-9 to improve CRC follow-up.
\end{abstract}

Correspondence to: Dr Osamu Nishimura, Division of Disease Proteomics, Institute for Protein Research, Osaka University, Osaka 565-0871, Japan

E-mail: osamu_nishimura@protein.osaka-u.ac.jp

Key words: biomarker, galectin, colorectal cancer, plasma, ELISA

\section{Introduction}

Colorectal cancer (CRC) is a significant cause of morbidity and mortality worldwide. Incidence and mortality rates are increasing in many countries along with changes in diet, such as increasing consumption of high-fat foods. In CRC, carcinoembryonic antigen (CEA) and carbohydrate antigen 19-9 (CA19-9) have been used as tumor markers. In early stage CRC, their expression levels are too low to be used as 'screening' markers. However, they are used as reliable CRC markers, monitoring tumor progression, for follow-up after surgery and for evaluation of ongoing treatments such as chemotherapy and radiation therapy (here, we term this type of marker as a 'tumor progression' marker). The American Society for Clinical Oncology has recommended the use of CEA as a marker for CRC follow-up (1) and the US Food and Drug Administration has also approved it as a CRC marker (2). In addition to CEA, clinical usage of CA19-9 has been approved by the Japanese Ministry of Health, Labor and Welfare for insurance coverage. In most CRC patients for whom CEA or CA19-9 can be used, these marker values show resectability or clinical response, decreasing after curative surgery and increasing when tumor recurrence or metastasis occurs (3). Consequently, these antigens are indispensable tumor progression markers for CRC follow-up. However, a significant number of CRC patients do not show increases in these maker values. Furthermore, elevated levels sometimes result from factors other than the tumor. Thus, it is important to develop additional complementary markers to achieve more comprehensive follow-up of the tumor, as well as one or more screening markers.

Great efforts have been dedicated to the identification of sensitive and specific CRC markers (4). Still, no screening marker and only CEA and CA19-9 as tumor progression markers have been clinically used in $\mathrm{CRC}$, even though many marker candidates have been reported. To identify potential CRC markers, we previously performed a quantitative proteomic analysis of human colon tissue samples, using the NBS (2-nitrobenzenesulfenyl) method (5), and identified 
many proteins that were differentially expressed between CRC tissues and non-cancer tissues (6). Among them, six proteins including galectin-1 were confirmed by IHC staining to be specifically elevated in cancer tissue (6). If a protein overexpressed in cancer tissue leaks into the peripheral circulation, it might be a tumor marker candidate. We focused on galectin-1 first, because it is mainly localized in extracellular spaces around cancer cells, in both early and advanced stages, and might be expected to easily leak into blood.

Galectins are a family of animal lectins that bind to $\beta$-galactosides through conserved carbohydrate recognition domains (CRD) (7). To date, 15 mammalian galectins have been identified and characterized. They are expressed both inside and outside cells and play important roles in a variety of cellular biological processes (8-11), including various stages of tumor progression and/or malignancy $(12,13)$. Galectin-1, galectin-3, and galectin-4 appear to be involved in the early development of human colon carcinoma (14) and the malignant transformation of the tumor (15). Especially, galectin-1 and galectin-3 play a role in the regulation of cell migration (16). In addition, the level of plasma galectin-1 is significantly up-regulated in patients with head and neck carcinomas compared to that found in healthy volunteers, and it is significantly decreased after therapeutic treatment (17). Galectin-7 plays positive and negative roles as a regulator of tumor progression, depending on the histological type of the tumor (18). Galectin-8 expression correlates negatively with the development of colon cancer malignancy and this protein is believed to suppress cancer cell migration (19).

Based on our previous studies (6) and other reports described above, we hypothesized that quantitating the level of circulating galectin proteins could provide valuable insight into the presence of cancers and the state of their progression. Therefore, we developed an ELISA measurement system for galectin proteins and measured the plasma levels to validate the use of galectins as CRC markers.

\section{Materials and methods}

Clinical data. Clinical information pertaining to the plasma samples used in this study is summarized in Table I. Blood samples $(20 \mathrm{ml})$ were taken twice from 105 CRC patients by venipuncture before intervention on the day of surgery (pre-surgical samples). Post-surgical blood samples were taken from 56 patients two weeks following surgery. The plasma levels of biomarkers for inflammatory responses in post-surgical samples, such as C-reactive protein and white blood cells, were confirmed to be within normal ranges. For control samples, we obtained 100 blood samples from healthy donors whose plasma levels of all six tumor makers (CEA, CA19-9, SCC, AFP, CA125, plus PSA for males and CA15-3 for females) were within normal ranges. Healthy individuals were confirmed to be free from any malignancies for at least two years following the day of venipuncture. Plasma was prepared by centrifugation of peripheral blood at 2,500 rpm for $25 \mathrm{~min}$ and stored at $-80^{\circ} \mathrm{C}$ until use.

In this study, both genders and their stages (based on UICC TNM Classification of Malignant Tumors) (20) were representative of the general population of CRC patients. They included $63(60.0 \%)$ males and 42 (40.0\%) females and
Table I. Summary of the clinical data of patients from whom plasma samples were obtained.

\begin{tabular}{lcc}
\hline & $\begin{array}{c}\text { CRC patients } \\
(\mathrm{n}=105)\end{array}$ & $\begin{array}{c}\text { Healthy donors } \\
(\mathrm{n}=100)\end{array}$ \\
\hline Age & & \\
Average \pm SD (range) $)$ & $63.5 \pm 11.3(28-88)$ & $61.2 \pm 9.9(40-86)$ \\
Gender & & 60 \\
Male & 63 & 40 \\
Female & 42 & \\
Patient characteristics & & \\
TNM classification & & \\
Stage 0 & 6 & \\
Stage I & 28 & \\
Stage II & 25 & \\
Stage III & 27 & \\
Stage IV & 19 & \\
Cure & & \\
A & 86 & \\
B & 8 & \\
C & 11 & \\
\hline
\end{tabular}

their mean age was 63.5 years old, ranging from 28 to 88 . The pathological stages of CRC patients were as follows: stage $0(n=6,5.7 \%)$, stage I $(n=28,26.7 \%)$, stage II $(n=25,23.8 \%)$, stage III $(\mathrm{n}=27,25.7 \%)$, and stage IV $(\mathrm{n}=19,18.1 \%)$. Their curability of the surgical treatment were classified into cure A $(\mathrm{n}=86,81.9 \%)$, cure $\mathrm{B}(\mathrm{n}=8,7.6 \%)$ and cure $\mathrm{C}(\mathrm{n}=11,10.5 \%)$. This study was approved by the Institutional Review Board of Osaka University, and informed consent was obtained from each patient.

Sandwich ELISA construction and measurement of galectin family proteins. Microtiter plates used for ELISA were prepared by using the IMMUNO-TEK ELISA Construction System (ZeptoMetrix, Buffalo, NY) according to the manufacturer's protocol. All 96 wells of the transparent plates (Maxisorp) were coated with $100 \mu 1$ of a capture antibody at the concentration of $5 \mu \mathrm{g} / \mathrm{ml}$. Each plasma sample was diluted to 10 or $20 \%$ in TBST buffer ( $20 \mathrm{mM}$ Tris- $\mathrm{HCl}$ (pH 7.4), $400 \mathrm{mM} \mathrm{NaCl}, 0.1 \%$ Tween-20), or further diluted when the concentration of the target protein was much higher. These diluted plasma samples as well as a dilution series of standard proteins were added to the plate wells (100 $\mu \mathrm{l} /$ well) and incubated for $1 \mathrm{~h}$ at room temperature. After washing the wells with TBST six times, $100 \mu 1$ of detection antibodies diluted in TBST were added and incubated for $1 \mathrm{~h}$ at room temperature. After washing the wells with TBST six times, either alkaline phosphatase (ALP) substrate or horseradish peroxidase (HRP) chromogene was added to detect the target protein.

Purified proteins and antibodies used for ELISA are listed in Table II. For the detection of galectin-1, the detection antibody was conjugated with ALP, and colorimetric ALP 
Table II. Purified proteins and antibodies used for ELISA construction.

\begin{tabular}{lccccc}
\hline & Galectin-1 ELISA & Galectin-2 ELISA & Galectin-3 ELISA & Galectin-4 ELISA & Galectin-7 ELISA \\
\hline $\begin{array}{l}\text { Recombinant } \\
\text { protein }\end{array}$ & $\begin{array}{c}\text { Recombinant } \\
\text { galectin-1 }\end{array}$ & $\begin{array}{c}\text { Recombinant } \\
\text { (Abnova, Taiwan) }\end{array}$ & $\begin{array}{c}\text { Recombinant } \\
\text { (R\&D Systems, } \\
\text { galectin-3 }\end{array}$ & $\begin{array}{c}\text { Recombinant } \\
\text { galectin-4 }\end{array}$ & $\begin{array}{c}\text { Recombinant } \\
\text { (R\&D Systems) } \\
\text { (R\&D Systems) }\end{array}$ \\
(R\&D Systems)
\end{tabular}

Purified proteins were used for calibration standards. Capture antibodies and detection antibodies were used at the concentration shown as above. Detection antibodies were conjugated with either alkaline phosphatase 'Alkaline Phosphatase Labeling Kit-NH2', Dojindo Molecular Technologies, Kumamoto, Japan), or horseradish peroxidase ('Peroxidase Labeling Kit-SH', Dojindo Molecular Technologies) according to the kit procedure.

substrate ('AP-Blue SpectraFX Microwell and/or Membrane Substrate'; BioFX Laboratories, Owings Mills, MD) was used to measure the absorbance at $595 \mathrm{~nm}$. For the detection of galectin-3 and galectin-4, the detection antibodies were conjugated with peroxidase, and HRP chromogene ('TMB Two Component HRP Microwell Substrate', BioFX Laboratories) was used to measure the absorbance at $450 \mathrm{~nm}$ and $595 \mathrm{~nm}$, while $595 \mathrm{~nm}$ was used as a reference. For the detection of galectin-2 and galectin-7, the detection antibody was conjugated with ALP, and chemiluminescent ALP substrate ('Chemiluminescent Ultra Sensitive AP Microwell and/or Membrane Substrate', BioFX Laboratories) was used to measure the luminescence. Color development and chemiluminescent reactions were performed according to the manufacturer's protocols. All the ELISA measurements for galectin family proteins were performed by Tecan GENios (Tecan Group Ltd., Zurich, Switzerland).

ELISA for CEA and CA19-9. Plasma concentrations of CEA were measured by The UniCel DxI (Beckman Coulter, Brea, CA) using a commercially available ELISA kit ('Access CEA'; Wako Pure Chemical Industries, Osaka, Japan). Those of CA19-9 were measured by SphereLight 180 (Olympus, Tokyo, Japan) using a commercially available ELISA kit ('SphereLight CA19-9'; Wako Pure Chemical Industries). Cut-off limits were employed from the manufacturer's instructions (CEA: $5 \mathrm{ng} / \mathrm{ml}, \mathrm{CA} 19-9: 37 \mathrm{U} / \mathrm{ml}$ ), and the samples with concentrations higher than these limits were judged to be positive.

Data analysis. Plasma concentrations of each target protein were compared among TNM-classified stages or other categorical groups, and the significance of differences was tested by various non-parametric methods such as the Mann-Whitney test, the Kruskall-Wallis with Dunn's post test, and the Wilcoxon matched pairs test. Spearman rank correlation test was performed to evaluate the correlation between tumor stages and plasma galectin concentrations. A receiver-operating characteristic (ROC) curve was plotted to determine the cut-off value for each galectin. All statistical analyses and graphical drawings were performed using JMP software version 8 (SAS Institute, Cary, NC), except for the Kruskall-Wallis with Dunn's post test (Prism 5 for Windows; GraphPad Software, San Diego, CA). All the tests were twotailed and their significance levels $(\alpha)$ were set at $\mathrm{P}<0.05$.

Immunohistochemical staining of galectin-1 and galectin-4. Detailed procedures for immunohistochemical (IHC) staining were described previously (6). The following antibodies were used: for primary antibodies, rabbit anti-human galectin-1 polyclonal antibody (Abcam, Cambridge, UK) and goat anti-human galectin-4 polyclonal antibody (R\&D Systems); for secondary antibodies, biotinylated sheep anti-rabbit IgG (Nichirei, Tokyo, Japan) for galectin-1 and biotinylated rabbit anti-goat $\mathrm{IgG}$ (Vector Laboratories, Burlingame, CA) for galectin-4. For negative control, the serial sections were tested using pre-immune rabbit (or goat) serum instead of each primary antibody. Tissue sections of CRC, which were found strongly positive in our previous study (6), were prepared as positive controls. All staining results were evaluated by a pathologist who was not given sample identities. For each IHC analysis, the mean intensity of the staining of tumor cells or stromal cells was evaluated in comparison with the positive controls as either weak, moderate, or strong. 
Table III. Spike-recovery test and cross-reactivity test for galectin ELISA systems constructed in this study.

A, Spike recovery test

\begin{tabular}{lccccc}
\hline & Galectin-1 & Galectin-2 & Galectin-3 & Galectin-4 & Galectin-7 \\
\hline Recovery rate $(\%)$ & 108.0 & 97.1 & 89.8 & 86.3 & 84.4 \\
\hline
\end{tabular}

B, Cross-reactivity test

\begin{tabular}{lccccc}
\hline $\begin{array}{l}\text { Cross-reactivity } \\
\text { rate (\%) with }\end{array}$ & $\begin{array}{c}\text { Recombinant } \\
\text { human galectin-1 }\end{array}$ & $\begin{array}{c}\text { Recombinant } \\
\text { human galectin-2 }\end{array}$ & $\begin{array}{c}\text { Recombinant } \\
\text { human galectin-3 }\end{array}$ & $\begin{array}{c}\text { Recombinant } \\
\text { human galectin-4 }\end{array}$ & $\begin{array}{c}\text { Recombinant } \\
\text { human galectin-7 }\end{array}$ \\
\hline Galectin-1 ELISA & n.a. & 0 & 0 & 0 & 0 \\
Galectin-2 ELISA & 1.370 & n.a. & 0 & 0 & 0 \\
Galectin-3 ELISA & 0 & 0 & n.a. & 0 & 0 \\
Galectin-4 ELISA & 0.518 & 0.478 & 0.397 & n.a. & 0.345 \\
Galectin-7 ELISA & 0 & 0 & 0 & 0 & n.a.
\end{tabular}

For spike recovery tests, plasma samples were spiked with known quantities of the corresponding recombinant human galectin protein. The recovery rate was the mean value of three sample measurements, where each recovery rate was calculated by comparing the observed value with the expected concentrations (A). For cross-reactivity tests, the other recombinant human galectins were diluted and directly tested in the ELISA system. The extent of cross-reactivity was determined by dividing the observed value by the applied protein concentration (B). n.a., not applicable.

\section{Results}

Validation of ELISA system constructed and used in this study. To measure the concentration of each galectin in human plasma specimens, we constructed a specific sandwich ELISA for each of the five galectins (galectin-1/-2/-3/-4/-7), for which both antibodies and purified proteins were commercially available. For all the galectins tested, ELISA systems were constructed and the standard curves were fitted from the measurement of serial dilutions of each recombinant galectin protein. The fitted ranges were as follows: $3.13-100 \mathrm{ng} / \mathrm{ml}$ for galectin-1, 0.0313-4.00 ng/ml for galectin-2, 0.156-20.0 ng/ml for galectin-3, 0.0313-1.00 ng/ml for galectin-4, and 0.625 $80.0 \mathrm{ng} / \mathrm{ml}$ for galectin-7 (data not shown). Plasma samples were diluted before measurement so that the measured values fell within the above ranges. In this study, the detection limit of each ELISA was set to 'the mean value plus twice the SD value' of multiple blank ( $>5$ wells) measurements: galectin-1, $2.91 \mathrm{ng} / \mathrm{ml}$; galectin-2, $0.0144 \mathrm{ng} / \mathrm{ml}$; galectin-3, $0.354 \mathrm{ng} / \mathrm{ml}$; galectin-4, $0.00362 \mathrm{ng} / \mathrm{ml}$; galectin-7, $0.155 \mathrm{ng} / \mathrm{ml}$. If a measured value was under each 'detection limit', the value was then defined as ' 0 '. All the CVs of intra-assays and interassays were within $7.80 \%$ and within $18.9 \%$, respectively (data not shown). Thus, reproducibility of our ELISA system was acceptable. Recovery rates from spiked samples were between 84.4 ad 108\%, whereas cross-reactivities for the other galectin family proteins were all within $1.37 \%$ (Table III). These results demonstrate that the ELISA system for each galectin family protein was accurate and specific.

Comparison of plasma galectin concentrations from CRC patients and healthy volunteers. Concentrations of galectin family proteins (galectin-1/-2/-3/-4/-7) in plasma samples from
$105 \mathrm{CRC}$ patients and 100 healthy volunteers (control) were determined by sandwich ELISA constructed and validated as mentioned above. We found that plasma concentrations for galectin-1/-3/-4 were significantly increased $(P=0.0001$, $\mathrm{P}=0.0003$, and $\mathrm{P}<0.0001$, respectively) in the CRC patient group compared to the healthy control group (Fig. 1), while there was no significant difference between the two groups for galectin-2 and -7 (data not shown). More detailed analyses were performed for galectin-1/-3/-4 as the plasma levels were significantly different between healthy controls and CRC patients.

Relationship between TNM classification and plasma galectin concentrations. CRC patients were classified by their tumor stage into three groups (stage 0, stages I and II, and stages III and IV) and we compared the plasma concentrations among the groups, including a healthy control group. Statistical analysis revealed that significant differences among the groups were present for all three galectins $(\mathrm{P}<0.01$; Kruskal-Wallis test, data not shown). Post-hoc analysis (Dunn's post test) was performed to find paired groups with significant differences. Although significant differences were not observed between the control group and the stage 0 group for any of the galectins, the medians of stage 0 were higher than those of the control for all three galectins (Fig. 2). In addition, all three galectin levels in stage I-II and stage III-IV groups were significantly higher $(\mathrm{P}<0.05)$ than those in the control group. Median and both quartiles of galectin-4 levels were elevated as the tumor stage progressed (Fig. 2C), while such a clear relationship for galectin-1 and galectin-3 was not observed (Fig. 2A and B). In fact, there was a significant correlation between galectin-4 levels and tumor stage (Spearman's rank correlation test: $r=0.344, \mathrm{P}=0.0009$ ). Because the levels of a 
(A)

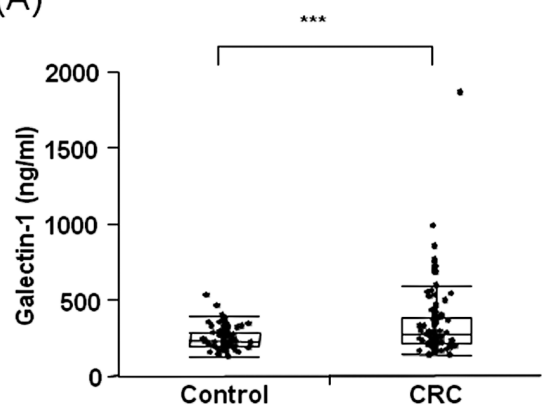

(B)

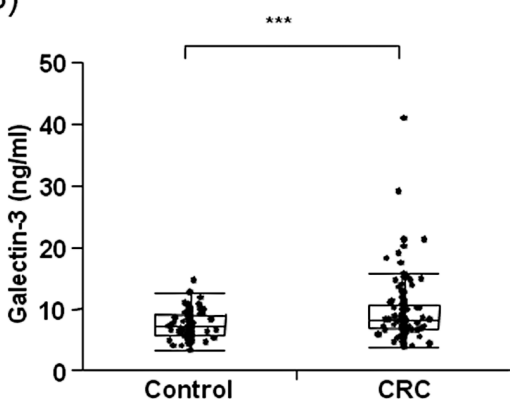

(C)

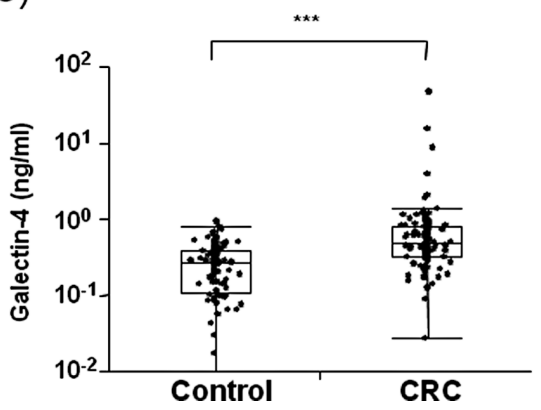

Figure 1. Plasma galectin levels in healthy volunteers (control) and CRC patients. Plasma concentrations of all individuals are plotted for galectin-1 (A), galectin-3 (B), and galectin-4 (C). The ends of the boxes indicate the upper and lower quartiles and the lines across the middle of the boxes indicate the median values in each case. The whiskers indicate the outermost data point, from the ends of the box, that falls within the distances calculated as follows: upper (or lower) quartile plus (or minus) 1.5-times the interquartile range. For galectin-1, the median and interquartile ranges of healthy controls were $236.7 \mathrm{ng} / \mathrm{ml}$ and 199.3-285.9 ng/ml, and those of CRC patients were $277.7 \mathrm{ng} / \mathrm{ml}$ and $224.6-392.4 \mathrm{ng} / \mathrm{ml}$. For galectin-3, the median and interquartile ranges of healthy controls were $7.16 \mathrm{ng} / \mathrm{ml}$ and $5.940-9.069 \mathrm{ng} / \mathrm{ml}$ and those of CRC patients were $8.31 \mathrm{ng} / \mathrm{ml}$ and $6.897-10.88 \mathrm{ng} / \mathrm{ml}$. For galectin- 4 , the median and interquartile ranges of healthy controls were $0.271 \mathrm{ng} / \mathrm{ml}$ and $0.1082-0.3952 \mathrm{ng} / \mathrm{ml}$ and those of CRC patients were $0.494 \mathrm{ng} / \mathrm{ml}$ and $0.3212-0.8226 \mathrm{ng} / \mathrm{ml}$. P-values indicating the significance of difference between controls and CRC patients were calculated by the Mann-Whitney test and are represented by the number of asterisks: $* * * \mathrm{P}<0.001, * * \mathrm{P}<0.01, * \mathrm{P}<0.05$. It should be noted that only the galectin-4 level was plotted exponentially along the y-axis.

(A)

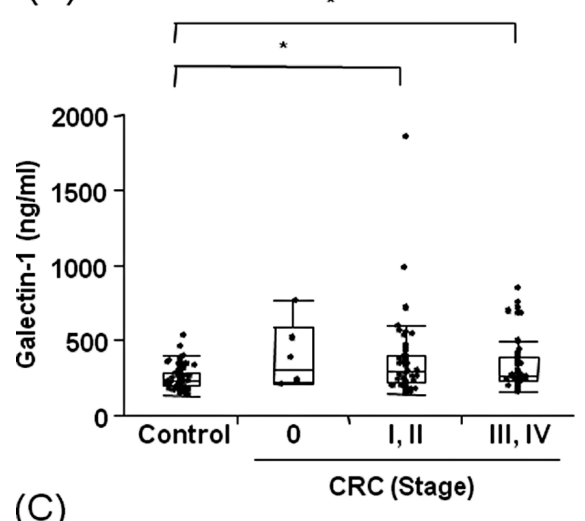

(C)

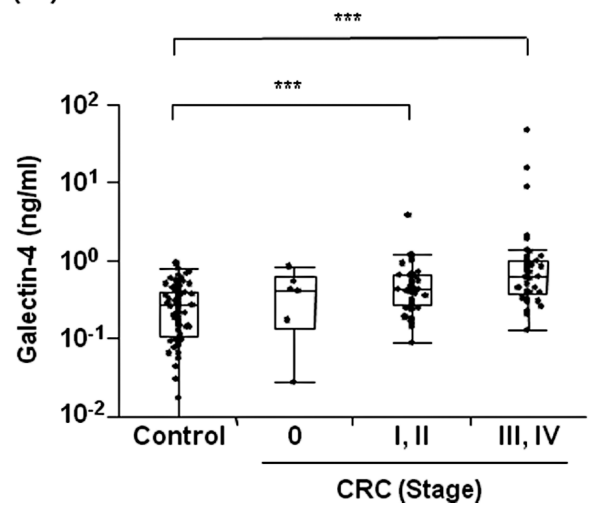

(B)

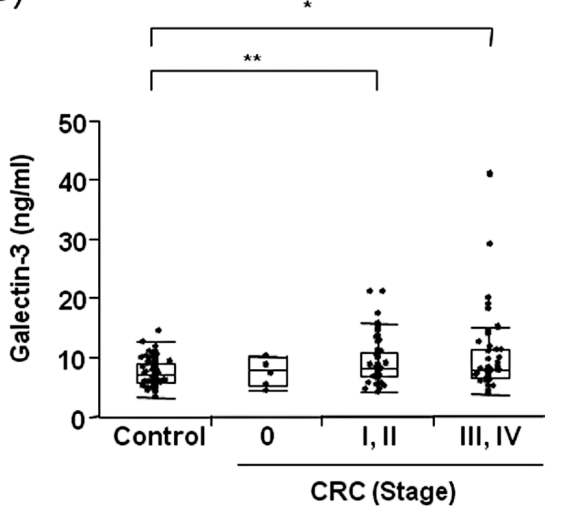

Figure 2. Comparison of the plasma galectin levels in CRC patients across tumor stages. Plasma concentrations for three groups of CRC patients as well as those for healthy controls are plotted for galectin-1 (A), galectin-3 (B), and galectin-4 (C). The Kruskall-Wallis test was performed to find a significant difference across the four groups. P-values of two particular groups were calculated by Dunn's post test. Only pairs with significant differences are indicated. Bar graphs are drawn as in Fig. 1. 
(A)

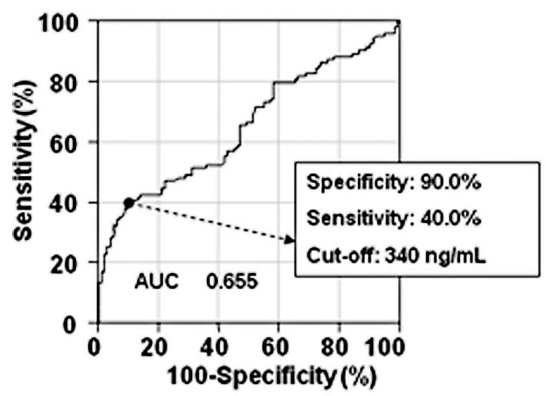

(C)

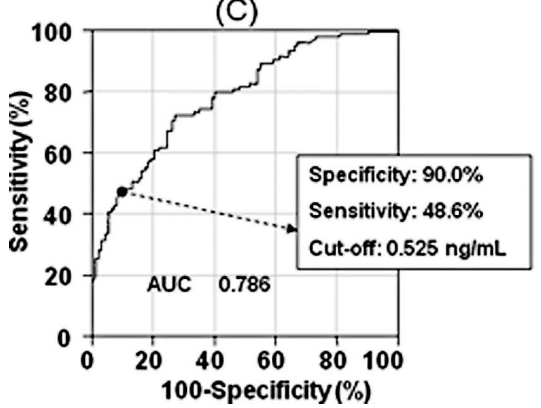

(A)

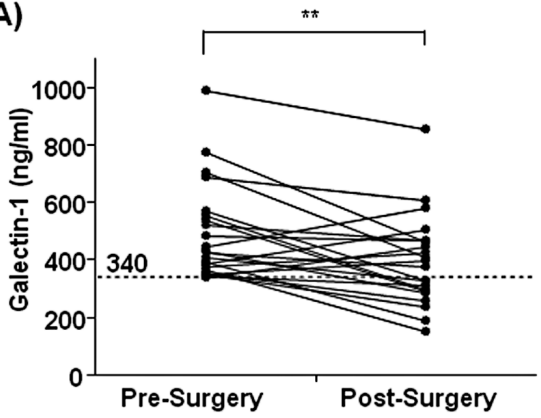

(C)

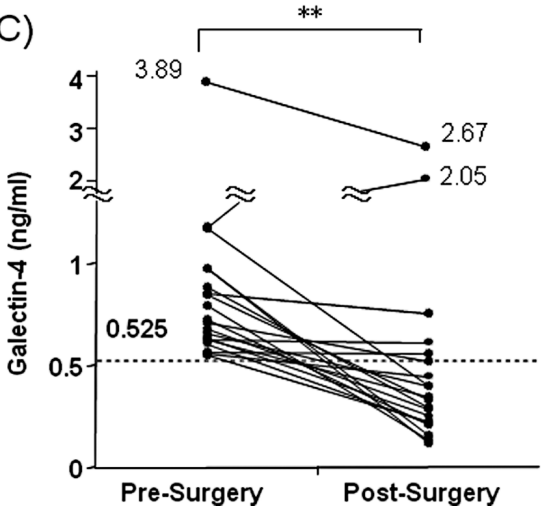

(B)

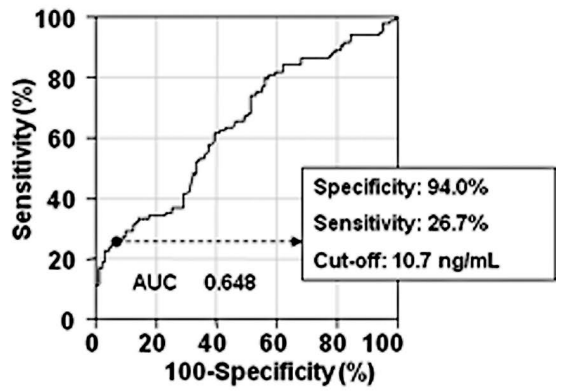

Figure 3. Receiver operating characteristic (ROC) curves of galectins for CRC detection. ROC curves of galectin-1 (A), galectin-3 (B), and galectin-4 (C) represent the relationship between sensitivity and specificity. Area under curve (AUC) is calculated as 0.655 for galectin-1, 0.648 for galectin-3, and 0.786 for galectin- 4 .

(B)

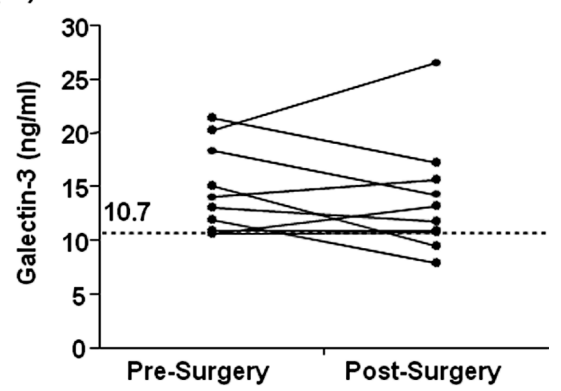

Figure 4. Plasma galectin levels in CRC patients before and after surgery. Plasma concentrations before (pre-) and after (post-) surgery for each patient are plotted for galectin-1 (A), galectin-3 (B), and galectin-4 (C). P-value between pre- and post-surgical values was calculated by Wilcoxon matched pairs test: $\mathrm{P}=0.0078$ for galectin- $1, \mathrm{P}=0.5417$ for galectin-3, and $\mathrm{P}=0.0045$ for galectin-4. 'tumor progression' marker properly reflect the stage of the tumor, galectin-4 belongs to this type of marker.

Determination of cut-off values for CRC detection. To determine the optimal cut-off value of each galectin for CRC detection, a receiver-operating characteristic (ROC) curve was drawn using plasma galectin levels of 105 CRC patients and 100 healthy controls (Fig. 3). For this purpose, the Youden Index (21) was introduced and its maximum point was calculated. However, cut-off values directly selected by this method showed high sensitivity but low specificity (data not shown), which is unacceptable for clinical use. Therefore, we put emphasis more on specificity in determining cut-off values and chose the maximum point of the Youden Index with high specificity $(>85 \%)$. Using this criterion, cut-off value for each galectin was set as 
(A)

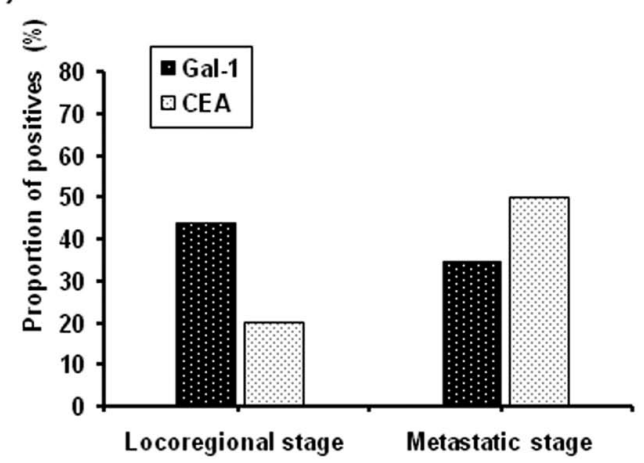

(C)

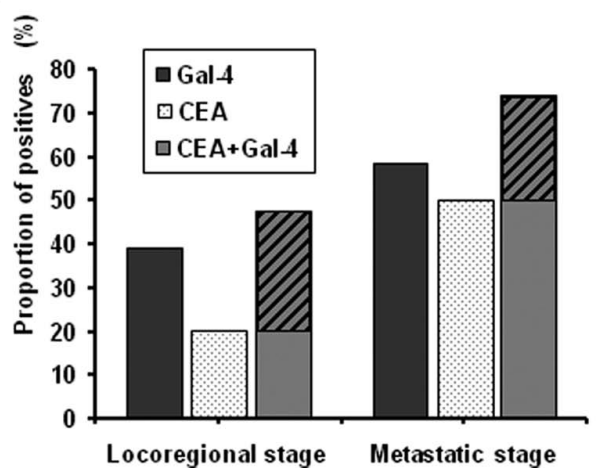

(B)

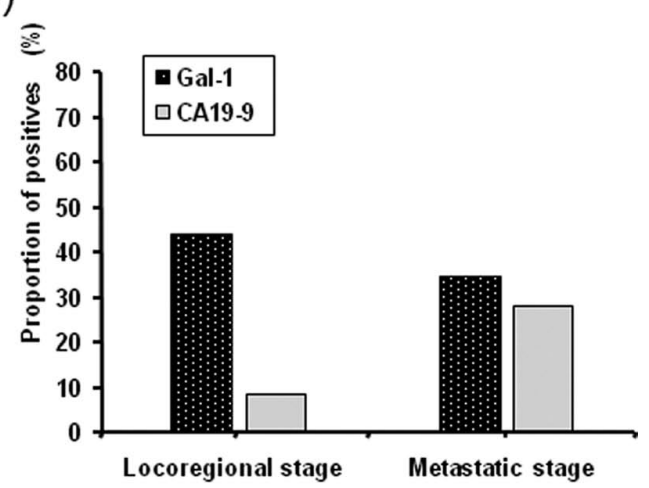

(D)

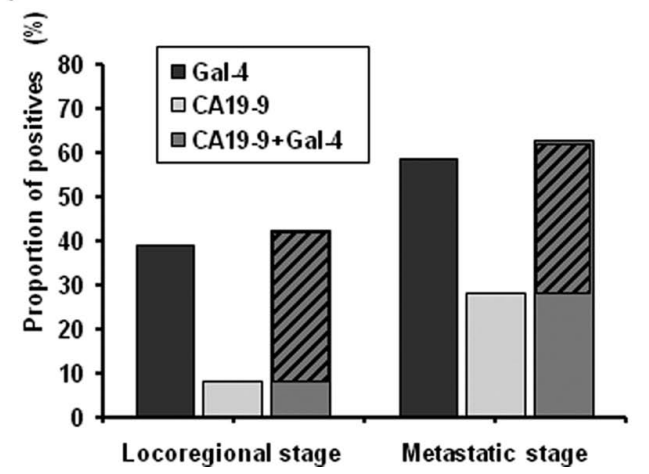

Figure 5. Proportion of patients positive for galectin-1, galectin-4, CEA, CA19-9, and their combinations in CRC patients with locoregional or metastatic disease. Percent of positives for each marker in the two groups of CRC patients was calculated from cut-off values: galectin-1, 340 ng/ml; galectin-4, $0.525 \mathrm{ng} / \mathrm{ml}$; CEA, $5 \mathrm{ng} / \mathrm{ml}$; CA19-9, $37 \mathrm{U} / \mathrm{ml}$. CRC marker candidates found in this study, galectin-1 (Gal-1; A and B) and galectin-4 (Gal-4; C and D), are compared with established CRC markers, CEA (A and C) and CA19-9 (B and D). CRC detection by dual markers, galection-4 and CEA (C) or galectin-4 and CA19-9 (D), are also evaluated. The increase in the percent of positives by the addition of galectin-4 marker is represented by dashed boxes.

follows: galecitin-1, $340 \mathrm{ng} / \mathrm{ml}$ with $90.0 \%$ specificity and $40.0 \%$ sensitivity; galectin-3, $10.7 \mathrm{ng} / \mathrm{ml}$ with $94.0 \%$ specificity and $26.7 \%$ sensitivity; and, galectin- $4,0.525 \mathrm{ng} / \mathrm{ml}$ with $90.0 \%$ specificity and $48.6 \%$ sensitivity. These values are appropriate for clinical use, because specificity at each of these cut-off values is similar to those for CEA or CA19-9 [for example, 90\% for CEA, (18); 93\% for CA19-9, (22)]. In this study, analyses hereafter were performed using these cut-off values.

Comparison of plasma galectin levels before and after surgery. Next, we investigated the level of each galectin in plasma before and after curative resection of the tumor. Samples of cure C patients were omitted, because they retained cancer after surgery and thus tumor marker values were expected not to decrease enough in post-surgical plasma. Matched pair samples of the 50 patients with both cure A and cure $\mathrm{B}$, whose plasma concentrations before surgery were higher than each cut-off value, were subjected to this analysis: 22 patients for galectin-1 (>340 ng/ml), 10 patients for galectin-3 (>10.7 ng/ml), and 20 patients for galectin-4 (>0.525 $\mathrm{ng} / \mathrm{ml})$. Plasma levels of both galectin-1 and galectin-4 were significantly decreased $(\mathrm{P}<0.01)$ after surgery (median for galectin-1 from 429 to 387 and that for galectin- 4 from 0.716 to 0.339 ), while the galectin-3 level was not altered (Fig. 4). The changes in galectin-4 levels were prominent such that most $(75.0 \% ; 15 / 20)$ of the post-surgical values fell below the cut-off values. These properties are requisite characteristics of tumor progression markers.

Galectin-4 CRC marker is complementary with CEA and/ or CA19-9: relationship between the proportion of positives and tumor stages in CRC patients. Combining galectin-3 with galectin-1 or galectin-4 hardly increased the proportion of positives in CRC patients (data not shown). Taken together with the results in the preceding section, the clinical utility of galectin-1 or galectin-4 as a plasma CRC marker is superior to that of galectin-3. Therefore, we focused on the former two galectins as CRC marker candidates and examined in detail the proportion of positives in CRC patients. Here, CRC patients were classified into two groups: those in a relatively early 'locoregional stage' including stages 0 , I, and II and those in a relatively late 'metastatic stage' including stages III and IV. The proportion of galectin-1 positives at the locoregional stage was relatively high $(44.1 \%$; 26/59), but did not increase at the metastatic stage $(34.8 \%$; 16/46) (Fig. 5A and B). High levels of positives at the early stage are advantageous for a 'screening' marker, the purpose of which is early detection of disease. On the other hand, the percentage of patients positive for galectin-4 increased along with the tumor stage progression (Fig. 5C and D; 39.0\% (23/59) in the locoregional stage and $58.7 \%$ (27/46) in metastatic stage), as is the case with CEA or CA19-9 (Fig. 5). Again, this property of galectin-4 is a characteristic of a tumor progression marker. 

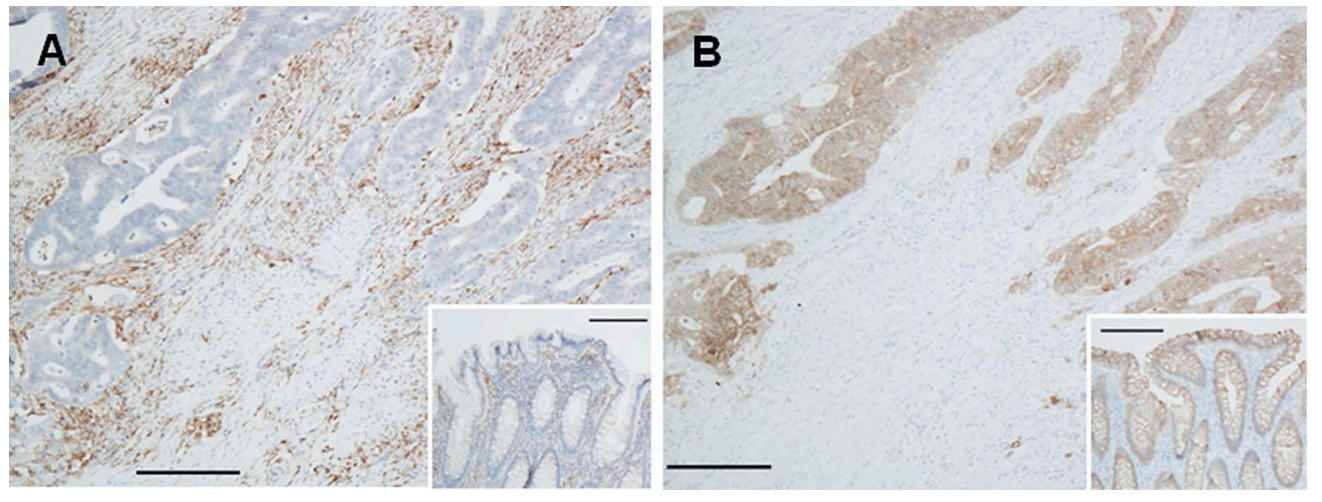

Figure 6. Immunohistochemical (IHC) staining of galectin-1 and galectin-4 in CRC tissue samples. IHC results using CRC tissues are shown for galectin-1 (A) and galectin-4 (B). Insets show IHC staining of normal colonic tissues. Bars indicate $200 \mu \mathrm{m}$ and the original magnification was $\mathrm{x} 50$, including insets. Each picture is a typical example of sixteen tested samples.

Detailed studies strongly suggest that galectin-4 has the characteristics of a tumor progression marker (Figs. 2C, $4 \mathrm{C}$ and $5 \mathrm{C}$ and $\mathrm{D})$. These results prompted us to examine the relationship between galectin-4 and typical CRC tumor progression markers, CEA or CA19-9. CEA is a reliable CRC marker used for follow-up. However, it unfortunately had a low percentage of positivity in CRC patients: for example, in this study, 20.3\% (12/59) in the locoregional stage and 50.0\% (23/46) in the metastatic stage (Fig. 5C). Combined use with galectin-4 much improved this percentage to $47.5 \%$ (28/59) for the locoregional stage and $73.9 \%$ (34/46) for the metastatic stage (Fig. 5C). Such an improvement by galectin- 4 was also observed for CA19-9; from $8.47 \%$ (5/59) to $42.4 \%$ (25/59) in the locoregional stage and from $28.3 \%$ (13/46) to $63.0 \%$ (29/46) in the metastatic stage (Fig. 5D).

Localization of galectin-1 and galectin-4 in normal and tumor tissue. Immunohistochemical staining was carried out to investigate the locations and expression patterns of galectin-1 and galectin-4 in CRC tissues (Fig. 6). Galectin-1 was primarily expressed in stromal cells, strongly in CRC tissues and weakly in normal colonic tissues. In contrast, galectin-4 was strongly expressed in cancer cells and moderately in normal epithelial cells, while no positive staining was detected in stroma.

\section{Discussion}

In our previous proteomic analysis of CRC tissue samples, galectin-1 was found to be a CRC-associated protein, increasing its expression in CRC tissues, a finding which was subsequently confirmed by IHC staining (6). Many studies have reported that the galectin family of proteins (including galectin-1) were related to tumor progression (12). Therefore, we hypothesized that the levels of circulating galectins could reflect the presence of CRC and/or the state of its progression. We constructed an ELISA system to assess their potential as plasma CRC markers. Among them, plasma levels of galectin-1 and galectin-4 were found to be significantly increased in CRC patients compared to healthy controls and their elevated levels significantly decreased after surgery. These results suggest that the levels of galectin-1 and galectin-4 in plasma reflect the presence of tumors or the reduction of tumor burden. That is, the plasma galectins are the consequence of cancer tissue leakage. In fact, elevated expression of galectin-1 in cancer tissue was previously confirmed by IHC staining (6). We carried out IHC staining for galectin-4 as well and found that this was also the case: expression of galectin-4 was elevated in cancer tissue (Fig. 6B). These observations are consistent with the previous report (14).

All the results with galectin- 4 presented in this study support the idea that galectin- 4 has the characteristics of a 'tumor progression' marker, such as CEA or CA19-9. The expression pattern of galectin-4, found in cancer cells but not in stroma, is also the same as that of CEA. As mentioned above, CEA is an indispensible and quite reliable CRC tumor progression marker which has been routinely used in clinical practice worldwide to follow up CRC patients after surgery. However, CEA is not a marker universal to all CRC patients, as it is not applicable when the marker value before surgery is equal to or lower than the cut-off value. In fact, only $44 \%$ of all CRC patients were CEA positive and only $33-45 \%$ were positive even in stage III where the tumor stage is rather advanced (22-25). In Fig. 5, we demonstrated that combined use of galectin-4 with CEA greatly increased the proportion of positive samples compared to the use of CEA alone. In addition, dual use of CEA and CA19-9 found that $36.2 \%$ of all CRC patients were positive, but the proportion of positives improved up to $60.0 \%$ when three markers were used (CEA, CA19-9, and galectin-4; Table IV). It is quite important for the level of a tumor progression marker to decrease below the cut-off value after curative resection of the tumor. In fact, this was the case for CEA in almost all CRC patients (3). In the case of galectin- 4 , most (75.0\%) of the patient plasma levels decreased below the cut-off value after surgery. These results demonstrate that galectin-4 has significant potential for use as a complementary CRC marker together with CEA. Another prominent feature of galectin- 4 is that a relatively high proportion of positives was found in CRC patients even at early stages of disease (Fig. $5 \mathrm{C}$ and D). This fact leads us to postulate that utilization of galectin- 4 marker could achieve early detection of recurrence/metastasis during post-surgical follow-up. 
Table IV. Multiparametric marker analysis

Sensitivity (\%)

\begin{tabular}{ll}
\hline CEA & 33.3 \\
CA19-9 & 17.1 \\
Galectin-4 & 47.6 \\
CEA + CA19-9 & $\mathbf{3 6 . 2}$ \\
CEA + Galectin-4 & 59.0 \\
CA19-9 + Galectin-4 & 51.4 \\
CEA + CA19-9 + Galectin-4 & $\mathbf{6 0 . 0}$
\end{tabular}

In multi-marker detection, patients who had a marker value larger than the cut-off value in either marker were judged as positives.

On the other hand, galectin-1 has features advantageous for a screening marker, because it showed a high percentage of positives in early stages (Fig. 5A and B). Evaluation of a potential screening marker needs far more samples and, in addition, demonstration of the tumor specificity is also requisite. Therefore, galectin-1 should be considered a 'candidate' screening marker until additional evaluations are completed. Nonetheless, our results with galectin-1 can be discussed further in terms of the relationship between marker type and localization/function. Galectin-1 is expressed in stroma around cancer cells (6) (Fig. 6A) and localizes mainly in the extracellular space. In addition, the expression level of galectin-1 is markedly elevated in early stages as well as in advanced stages, in contrast to those of galectin-4, CEA, and CA19-9 (data not shown). This pattern of expression and localization might make it easier for galectin-1 to reach the peripheral circulation and also for its plasma level to increase even in early stages of CRC. Galectin-1 expression appears to be involved in tumor cell escape from immune responses (25-28). Considering that plasma galectin-1 levels were elevated from early stages of $\mathrm{CRC}$, it is possible that galectin-1 has some relevance to tumor maintenance.

Recent evidence suggests that the interaction between tumor cells and surrounding stroma plays a critical role in tumor growth, invasion, metastasis, angiogenesis, and chemoresistance (29-34). It has been shown that galectin-1 regulates cell migration and that galectin-1 binding sites are displayed on cell surfaces in most colon cancer cell lines, while only one of them actually expresses galectin-1 (16). Taken together, galectin-1 expression by stroma may play a role in colon cancer cell migration via a paracrine mechanism.

It was also reported that changes occurring in tumor stroma correlate with disease outcome (35). However, few tumor markers derived from stroma have been identified in the plasma. In this study, we demonstrated the possibility that galectin-1 from tumor stroma might be useful as a plasma screening marker. Focusing on tumor stroma as well as cancer cells could enhance the possibility of discovering novel biomarkers.

This report describes our unique and interesting findings that the two galectins are two different types of plasma markers: galectin-1 could be used as a possible screening marker while galectin-4 may be used as a tumor progression marker. In addition, localization of their expression in cancer tissues was found to be complementary: galectin-1 expression was essentially limited to the stroma, whereas galectin-4 was specifically expressed in cancer cells (Fig. 6). As for their relevance to tumorigenesis, galectin-1 enhances tumor progression, while galectin-4 represses it (12). Biochemically, they bind and gather substrates with two 'hands' of carbohydrate recognition domains (CRDs), but they differ in their CRDs and their composition: galectin-1 with one CRD functions as a homodimer while galectin- 4 functions as a monomer with two distinct CRDs (12). Taken together, the mechanisms by which the two galectins function seem to be quite similar, but their functions and nature of the markers are quite different. We speculate that these differences arise from differences in their localization and/or their interaction partners. It is important to define these issues to more fully understand these important $\mathrm{CRC}$ marker proteins.

In this study, we focused on the evaluation of the galectin family of proteins with regard to their possible application as plasma markers. These proteins might be targets for therapeutic interventon, a subject which will be examined in the future.

\section{References}

1. Locker GY, Hamilton S, Harris JM, Jessup JM, Kemeny, N, Macdonald JS, Somerfield MR, Hayes DF and Bast RC Jr: ASCO 2006 update of recommendations for the use of tumor markers in gastrointestinal cancer. J Clin Oncol 24: 5313-5327, 2006.

2. Gutman S and Kessler LG: The US Food and Drug Administration perspective on cancer biomarker development. Nat Rev Cancer 6: 565-571, 2006.

3. Grossmann I, de Bock GH, Meershoek-Klein Kranenbarg WM, van de Velde CJ and Wiggers T: Carcinoembryonic antigen (CEA) measurement during follow-up for rectal carcinoma is useful even if normal levels exist before surgery. A retrospective study of CEA values in the TME trial. Eur J Surg Oncol 33: 183-187, 2007.

4. Kuramitsu Y and Nakamura K: Proteomic analysis of cancer tissues: shedding light on carcinogenesis and possible biomarkers. Proteomics 6: 5650-5661, 2006.

5. Matsuo E, Watanabe M, Kuyama $\mathrm{H}$ and Nishimura O: A new strategy for protein biomarker discovery utilizing 2-nitrobenzenesulfenyl (NBS) reagent and its applications to clinical samples. J Chromatogr B Analyt Technol Biomed Life Sci 877: 2607-2614, 2009

6. Watanabe M, Takemasa I, Kawaguchi N, Miyake M, Nishimura N, Matsubara T, Matsuo E, Sekimoto M, Nagai K, Matsuura N, Monden M and Nishimura O: An application of the 2-nitrobenzenesulfenyl method to proteomic profiling of human colorectal carcinoma: a novel approach for biomarker discovery. Proteomics Clin Appl 2: 925-935, 2008.

7. Kasai $\mathrm{K}$ and Hirabayashi J: Galectins: a family of animal lectins that decipher glycocodes. J Biochem 119: 1-8, 1996.

8. Yang RY and Liu FT: Galectins in cell growth and apoptosis. Cell Mol Life Sci 60: 267-276, 2003.

9. Dagher SF, Wang JL and Patterson RJ: Identification of galectin-3 as a factor in pre-mRNA splicing. Proc Natl Acad Sci USA 92: 1213-1217, 1995.

10. Wang JL, Gray RM, Haudek KC and Patterson RJ: Nucleocytoplasmic lectins. Biochim Biophys Acta 1673: 75-93, 2004.

11. Vyakarnam A, Dagher SF, Wang JL and Patterson RJ: Evidence for a role for galectin-1 in pre-mRNA splicing. Mol Cell Biol 17: 4730-4737, 1997.

12. Liu FT and Rabinovich GA: Galectins as modulators of tumour progression. Nat Rev Cancer 5: 29-41, 2005.

13. Danguy A, Camby I and Kiss R: Galectins and cancer. Biochim Biophys Acta 1572: 285-293, 2002. 
14. Nagy N, Legendre H, Engels O, Andre S, Kaltner H, Wasano K, Zick Y, Pector JC, Decaestecker C, Gabius HJ, Salmon I and Kiss R: Refined prognostic evaluation in colon carcinoma using immunohistochemical galectin fingerprinting. Cancer 97: 1849-1858, 2003.

15. Nakamura $\mathbf{M}$, Inufusa $\mathrm{H}$, Adachi $\mathrm{T}$, Aga $\mathrm{M}$, Kurimoto $\mathrm{M}$, Nakatani Y, Wakano T, Nakajima A, Hida JI, Miyake M, Shindo K and Yasutomi M: Involvement of galectin-3 expression in colorectal cancer progression and metastasis. Int $\mathrm{J}$ Oncol 15: 143-148, 1999.

16. Hittelet A, Legendre H, Nagy N, Bronckart Y, Pector JC, Salmon I, Yeaton P, Gabius HJ, Kiss R and Camby I: Upregulation of galectins-1 and -3 in human colon cancer and their role in regulating cell migration. Int J Cancer 103: 370-379, 2003.

17. Saussez S, Lorfevre F, Lequeux T, Laurent G, Chantrain GC, Vertongen F, Toubeau G, Decaestecker C and Kiss R: The determination of the levels of circulating galectin-1 and -3 in HNSCC patients could be used to monitor tumor progression and/or responses to therapy. Oral Oncology 44: 86-93, 2008.

18. Saussez S and Kiss R: Galectin-7. Cell Mol Life Sci 63: 686-697, 2006.

19. Nagy N, Bronckart Y, Camby I, Legendre H, Lahm H, Kaltner H, Hadari Y, Van Ham P, Yeaton P, Pector JC, Zick Y, Salmon I, Danguy A, Kiss R and Gabius HJ: Galectin-8 expression decreases in cancer compared with normal and dysplastic human colon tissue and acts significantly on human colon cancer cell migration as a suppressor. Gut 50: 392-401, 2002.

20. Sobin LH and Wittekind Ch (eds): TNM Classification of malignant tumors. 5th edition. John Wiley \& Sons, Inc., New York, 1997.

21. Youden WJ: Index for rating diagnostic tests. Cancer 3: 32-35, 1950.

22. Yamashita $\mathrm{K}$ and Watanabe M: Clinical significance of tumor markers and an emerging perspective on colorectal cancer. Cancer Sci 100: 195-199, 2009.

23. Herszenyi L, Farinati F, Cardin R, Istvan G, Molnar LD, Hritz I, De Paoli M, Plebani M and Tulassay Z: Tumor marker utility and prognostic relevance of cathepsin B, cathepsin L, urokinasetype plasminogen activator, plasminogen activator inhibitor type-1, CEA and CA 19-9 in colorectal cancer. BMC Cancer 8: $194,2008$.

24. Louhimo J, Carpelan-Holmstrom M, Alfthan H, Stenman UH, Jarvinen HJ and Haglund C: Serum HCG beta, CA 72-4 and CEA are independent prognostic factors in colorectal cancer. Int J Cancer 101: 545-548, 2002.

25. Rabinovich GA, Ariel A, Hershkoviz R, Hirabayashi J, Kasai KI and Lider O: Specific inhibition of T-cell adhesion to extracellular matrix and proinflammatory cytokine secretion by human recombinant galectin-1. Immunology 97: 100-106, 1999.
26. Chung CD, Patel VP, Moran M, Lewis LA and Miceli MC: Galectin-1 induces partial TCR zeta-chain phosphorylation and antagonizes processive TCR signal transduction. J Immunol 165: 3722-3729, 2000.

27. Rubinstein N, Alvarez M, Zwirner NW, Toscano MA, Ilarregui JM, Bravo A, Mordoh J, Fainboim L, Podhajcer OL and Rabinovich GA: Targeted inhibition of galectin-1 gene expression in tumor cells results in heightened $\mathrm{T}$ cell-mediated rejection; A potential mechanism of tumor-immune privilege. Cancer Cell 5: 241-251, 2004.

28. He $\mathrm{J}$ and Baum LG: Presentation of galectin-1 by extracellular matrix triggers $\mathrm{T}$ cell death. J Biol Chem 279: 4705-4712, 2004.

29. Gleave M, Hsieh JT, Gao CA, von Eschenbach AC and Chung LW: Acceleration of human prostate cancer growth in vivo by factors produced by prostate and bone fibroblasts. Cancer Res 51: 3753-3761, 1991.

30. Nakamura T, Matsumoto K, Kiritoshi A, Tano $\mathrm{Y}$ and Nakamura T: Induction of hepatocyte growth factor in fibroblasts by tumor-derived factors affects invasive growth of tumor cells: in vitro analysis of tumor-stromal interactions. Cancer Res 57: 3305-3313, 1997

31. Janvier R, Sourla A, Koutsilieris M and Doillon CJ : Stromal fibroblasts are required for PC-3 human prostate cancer cells to produce capillary-like formation of endothelial cells in a threedimensional co-culture system. Anticancer Res 17: 1551-1557, 1997.

32. Anderson IC, Mari SE, Broderick RJ, Mari BP and Shipp MA: The angiogenic factor interleukin 8 is induced in non-small cell lung cancer/pulmonary fibroblast cocultures. Cancer Res 60: 269-272, 2000

33. Bhowmick NA, Chytil A, Plieth D, Gorska AE, Dumont N, Shappell S, Washington MK, Neilson EG and Moses HL: TGF-beta signaling in fibroblasts modulates the oncogenic potential of adjacent epithelia. Science 303: 848-851, 2004.

34. Muerkoster S, Wegehenkel K, Arlt A, Witt M, Sipos B, Kruse ML, Sebens T, Kloppel G, Kalthoff H, Folsch UR and Schafer H: Tumor stroma interactions induce chemoresistance in pancreatic ductal carcinoma cells involving increased secretion and paracrine effects of nitric oxide and interleukin-1beta. Cancer Res 64: 1331-1337, 2004.

35. Finak G, Bertos N, Pepin F, Sadekova S, Souleimanova M, Zhao H, Chen H, Omeroglu G, Meterissian S, Omeroglu A, Hallett $\mathrm{M}$ and Park M: Stromal gene expression predicts clinical outcome in breast cancer. Nat Med 14: 518-527, 2008. 